PROCEEDINGS OF THE

AMERICAN MATHEMATICAL SOCIETY

Volume 139, Number 11, November 2011, Pages 4121-4135

S 0002-9939(2011)10821-9

Article electronically published on March 21, 2011

\title{
ON TOTALLY GEODESIC FOLIATIONS AND DOUBLY RULED SURFACES IN A COMPACT LIE GROUP
}

\author{
MARIUS MUNTEANU AND KRISTOPHER TAPP
}

(Communicated by Jianguo Cao)

\begin{abstract}
For a Riemannian submersion from a simple compact Lie group with a bi-invariant metric, we prove that the action of its holonomy group on the fibers is transitive. As a step towards classifying Riemannian submersions with totally geodesic fibers, we consider the parameterized surface induced by lifting a base geodesic to points along a geodesic in a fiber. Such a surface is "doubly ruled" (it is ruled by horizontal geodesics and also by vertical geodesics). Its characterizing properties allow us to define "doubly ruled parameterized surfaces" in any Riemannian manifold, independent of Riemannian submersions. We initiate a study of the doubly ruled parameterized surfaces in compact Lie groups and in other symmetric spaces by establishing several rigidity theorems and constructing examples.
\end{abstract}

\section{INTRODUCTION}

Riemannian submersions are a central tool for many geometric constructions such as nonnegatively curved manifolds and Einstein manifolds, but they are also important for proving rigidity theorems. For example, the proof of the Diameter Rigidity Theorem for positively curved manifolds 4 hinged on the classification of Riemannian submersions from round spheres (achieved in 5], 14]). This theorem motivated work towards classifying Riemannian submersions from other symmetric spaces. For example, the Riemannian submersions from flat Euclidean spaces were classified in [6]. Those from $\mathbb{C P}^{n}$ with totally geodesic fibers had been previously classified in 2].

K. Grove posed the problem of classifying Riemannian submersions from compact Lie groups with bi-invariant metrics [7, Problem 5.4]. The special case of onedimensional fibers was solved in [10]. The general case is likely to be difficult, especially in light of recent examples of infinite families which are not biquotient submersions 9]. We at least establish the following rigidity:

Proposition 1.1. If $G$ is a simple compact Lie group with bi-invariant metric and $\pi: G \rightarrow B$ is a Riemannian submersion, then the holonomy group of $\pi$ acts transitively on the fibers.

Another known rigidity phenomenon in this context is that the O'Neill tensor vanishes on horizontal zero-curvature planes [13. Grove's question appears more

Received by the editors June 12, 2010 and, in revised form, September 28, 2010.

2010 Mathematics Subject Classification. Primary 53C12.

Key words and phrases. Riemannian submersion, Lie group, good triple, doubly ruled surface.

(C)2011 American Mathematical Society 
tractable under the added assumption that the fibers are totally geodesic, in which case one expects:

Conjecture 1.2. If $G$ is a simple compact Lie group with bi-invariant metric and $\pi: G \rightarrow B$ is a Riemannian submersion with connected totally geodesic fibers, then it is a left or right coset fibration; that is, $B=G / H$ or $B=H \backslash G$ for some subgroup $H \subset G$, and $\pi$ is the quotient map.

If $G$ is not simple, the conjecture is still reasonable but must be reworded to allow for submersions such as $\pi: G_{1} \times G_{2} \rightarrow\left(H_{1} \backslash G_{1}\right) \times\left(G_{2} / H_{2}\right)$ (where $H_{i} \subset G_{i}$ ), which have totally geodesic fibers but are only equivariantly isometric to (not literally equal to) coset fibrations. The conjecture was proven in [1] if the fiber through the identity contains a maximal torus of $G$.

To classify Riemmanian submersions with totally geodesic fibers from any space $M$, we propose that it is useful to first understand the "good triples" of vectors, defined as follows:

Definition 1.3. Let $M$ be a Riemannian manifold and let $p \in M$. We refer to a triple $\{X, V, \mathcal{A}\} \subset T_{p} M$ as $\operatorname{good}$ if $\exp (t \cdot V(s))=\exp (s \cdot X(t))$ for all $s, t \in \mathbb{R}$, where $V(s)$ denotes the Jacobi field along $s \mapsto \exp (s X)$ with $V(0)=V$ and $V^{\prime}(0)=\mathcal{A}$ and where $X(t)$ denotes the Jacobi field along $t \mapsto \exp (t V)$ with $X(0)=X$ and $X^{\prime}(0)=$ $\mathcal{A}$. In this case, the parameterized surface $f(s, t)=\exp (t \cdot V(s))=\exp (s \cdot X(t))$ will be called a doubly ruled parameterized surface.

A "doubly ruled surface" in $M$ means a surface which admits a transversal pair of smooth foliations by geodesics of $M$. A "doubly ruled parameterized surface" is a special type of doubly ruled surface, namely, one whose geodesic foliations arise as the constant-s and constant- $t$ curves of some single parameterization $(s, t) \mapsto$ $f(s, t)$. We will construct examples in compact Lie groups beyond those which arise from coset fibrations via the following relationship between good triples and the submersion problem:

Proposition 1.4. If $\pi: M \rightarrow B$ is a Riemannian submersion with totally geodesic fibers, $p \in M$, and $X, V \in T_{p} M$ with $V$ vertical and $X$ horizontal, then $\{X, V, A(X, V)\}$ is a good triple (where $A$ denotes the O'Neill tensor).

The doubly ruled parameterized surface corresponding to $\{X, V, A(X, V)\}$ is the "vertizontal" surface induced by lifting a single geodesic in the base space to points along a geodesic in a fiber, so this good triple might be thought of as the infinitesimal version of a vertizontal surface.

Aside from its relevance to the submersion problem, the classification of doubly ruled parameterized surfaces in symmetric spaces is perhaps a problem of independent interest, related to the classical classification of doubly ruled surfaces in $\mathbb{R}^{3}$ (see [8]), and its many generalizations, for example, to doubly ruled submanifolds of space forms in [3]. The main purpose of this paper is to initiate the exploration of good triples (and hence doubly ruled parameterized surfaces) in symmetric spaces. For compact Lie groups, we characterize the good triples as follows:

Theorem 1.5. Let $G$ be a compact Lie group with a bi-invariant metric. Let $\mathfrak{g}$ denote the Lie algebra of $G$. The triple $\{X, V, \mathcal{A}\} \subset \mathfrak{g}$ is good if and only if for all integers $n, m \geq 0$, we have

$$
\left[\operatorname{ad}_{X}^{n} B, \operatorname{ad}_{V}^{m} \bar{B}\right]=0
$$

where $B=\frac{1}{2}[X, V]-\mathcal{A}$ and $\bar{B}=\frac{1}{2}[V, X]-\mathcal{A}$. 
Recall that the $A$-tensor of a left or right coset fibration is given by

$$
A(X, V)= \pm \frac{1}{2}[X, V]
$$

for all $X \in \mathfrak{g}$ horizontal and $V \in \mathfrak{g}$ vertical (plus for a left coset fibration and minus for right), so the condition is obviously satisfied for the triple $\{X, V, A(X, V)\}$.

Using Theorem 1.5 we establish several rigidity properties for good triples. For example, we prove that the Jacobi fields induced by a good triple (as in Definition 1.3) are of constant length, provided they are of bounded length. Additionally, we prove that given $\{X, V\}$, there are only finitely many choices for $\mathcal{A}$ for which $\{X, V, \mathcal{A}\}$ is good, provided that $X$ (or $V$ ) satisfies a generic property which we call "weak regularity."

We also exhibit several examples of good triples $\{X, V, \mathcal{A}\}$ in simple compact Lie groups which do not come from coset fibrations (because $\mathcal{A} \neq \pm \frac{1}{2}[X, V]$ ). One example, in which $X$ is not weakly regular, indicates that a full understanding of the good triples, even in a particular compact Lie group, can be subtle.

The $n=m=0$ case of the condition in Theorem 1.5 says that $[[X, V], \mathcal{A}]=0$. In the final section of the paper, we prove that the analogous equation holds in more general spaces:

Proposition 1.6. If $\{X, V, \mathcal{A}\}$ is a good triple in a locally symmetric space $M$, then $R(X, V) \mathcal{A}=0$, where $R$ denotes the curvature tensor of $M$.

\section{Transitive holOnOMY}

In this section, we prove Proposition 1.1 as an application of Wilking's dual foliations work (more specifically, Theorem 3b and Proposition 6.1 of [15]). The idea of the following proof is essentially found in [12].

Proof of Proposition 1.1. Let $G$ be a simple compact Lie group with a bi-invariant metric. Let $\mathfrak{g}$ denote its Lie algebra. Let $\pi: G \rightarrow B$ be a Riemannian submersion. Denote by $\mathcal{H}_{e}$ and $\mathcal{V}_{e}$ the horizontal and vertical spaces at the identity $e \in G$. Let $\tilde{H}$ denote the holonomy group of the submersion, which means the group of all diffeomorphisms of the fiber $\pi^{-1}(e)$ associated to piecewise-smooth loops in $B$ based at $\pi(e)$.

Let $\mathfrak{m} \subset \mathcal{V}_{e}$ denote the space of vertical vectors orthogonal to the holonomy orbit $\tilde{H} \star e$. It will suffice to prove that $\mathfrak{m}=\{0\}$.

Suppose there exists a nonzero vector $V \in \mathfrak{m}$. By Wilking's above-mentioned results, for all $X \in \mathcal{H}_{e}, \sigma=\operatorname{span}\{X, V\}$ is a zero-curvature plane, so $[X, V]=0$. Let $\alpha(t)=\exp (t X)$ and let $V(t)$ be the parallel field along $\alpha(t)$ with $V(0)=V$. Since $\sigma$ is tangent to a totally geodesic flat in $G, V(t)$ is both right and left invariant along $\alpha(t)$. In particular, $\operatorname{Ad}_{\alpha(t)} V=V$ for all $t$.

More generally, if $\alpha(t)$ is any horizontal piecewise geodesic path in $G$, then $\operatorname{Ad}_{\alpha(t)} V=V$ for all $t$. To see this, suppose that $\left\{\alpha\left(t_{1}\right), \alpha\left(t_{2}\right), \ldots\right\}$ are the nonsmooth points of $\alpha$. Let $V(t)$ be the parallel transport of $V$ along $\alpha(t)$. As shown in [15], $V(t)$ remains orthogonal to the holonomy orbits, so $\sec \left\{Y, V\left(t_{1}\right)\right\}=0$ for all horizontal vectors $Y$, particularly for the right-derivative $Y=\alpha^{\prime}\left(t_{1}^{+}\right)$. Then $\left.V\right|_{\left[t_{1}, t_{2}\right]}$ is left (respectively right) invariant along $\left.\alpha\right|_{\left[t_{1}, t_{2}\right]}$ because left (respectively right) multiplication by $\alpha\left(t_{1}\right)^{-1}$ sends it to a parallel field along a geodesic which exponentiates to a flat and is therefore left (respectively right) invariant. 
Therefore, $\operatorname{Ad}_{g} V=V$ for all $g \in \tilde{H} \star e$. It follows that $[Z, V]=0$ for all $Z \in \mathcal{V}_{e}$ tangent to $\tilde{H} \star e$. Thus, $\mathfrak{g}=\mathfrak{m} \oplus\left(\mathfrak{m}^{\perp}\right)$ is a decomposition of $\mathfrak{g}$ into commuting subspaces. It follows that $\mathfrak{m}$ and $\mathfrak{m}^{\perp}$ are both ideals of $\mathfrak{g}$, which contradicts the hypothesis that $G$ is a simple Lie group.

\section{The GEOMETRY OF RiemANNIAN SUBMERSIONS WITH TOTALLY GEODESIC FIBERS}

In this section, we summarize some facts about Riemannian submersions $\pi$ : $M \rightarrow B$ with totally geodesic fibers and prove Proposition 1.4 Let $p \in M$. Let $\mathcal{H}_{p}$ and $\mathcal{V}_{p}$ denote the horizontal and vertical spaces of $\pi$ at $p$.

We will describe how a pair $\{X, V\}$ (with $X \in \mathcal{H}_{p}$ and $V \in \mathcal{V}_{p}$ ) determines a parameterized surface in $M$. Let $t \mapsto \gamma(t)$ denote the vertical geodesic in $M$ with $\gamma(0)=p$ and $\gamma^{\prime}(0)=V$. Let $s \mapsto \alpha_{0}(s)$ denote the horizontal geodesic with $\alpha_{0}(0)=p$ and $\alpha_{0}^{\prime}(0)=X$. Notice that $s \mapsto \pi\left(\alpha_{0}(s)\right)$ is a geodesic in $B$. For each $t \in \mathbb{R}$, let $s \mapsto \alpha_{t}(s)$ denote the horizontal lift of $s \mapsto \pi\left(\alpha_{0}(s)\right)$ beginning at $\alpha_{t}(0)=\gamma(t)$. The parameterized surface $f(s, t):=\alpha_{t}(s)$ has several special properties, which we discuss next.

First, by definition, this surface is ruled by horizontal geodesics. Therefore, for any fixed $t_{0} \in \mathbb{R}$, the variational field $s \mapsto V_{t_{0}}(s):=\frac{\partial f}{\partial t}\left(s, t_{0}\right)$ is a vertical Jacobi field along the horizontal geodesic $s \mapsto f\left(s, t_{0}\right)=\alpha_{t_{0}}(s)$. These fields are often called "holonomy Jacobi fields" because they are related to the "holonomy isometries", $h_{s}: \pi^{-1}(\pi(p)) \rightarrow \pi^{-1}\left(\pi\left(\alpha_{0}(s)\right)\right)$ between the fibers. Recall that for $q \in \pi^{-1}(\pi(p))$, $h_{s}(q)$ is defined as the endpoint of the horizonal lift of $\left.\pi \circ \alpha_{0}\right|_{[0, s]}$ beginning at $q$. The holonomy Jacobi fields record the derivatives of the holonomy isometries; that is, $d\left(h_{s}\right)_{p}(V)=V_{0}(s)$. Since $h_{s}$ is an isometry, we learn that $V_{0}$ (and similarly each $\left.V_{t_{0}}\right)$ is a constant-length Jacobi field.

Second, this surface is also ruled by vertical geodesics. That is, for each $s_{0} \in \mathbb{R}$, the path $t \mapsto f\left(s_{0}, t\right)$ is a vertical geodesic because it is the image of the geodesic $t \mapsto \gamma(t)$ under the holonomy isometry $h_{s_{0}}$. Therefore, for any fixed $s_{0} \in \mathbb{R}$, the variational field $t \mapsto X_{s_{0}}(t):=\frac{\partial f}{\partial s}\left(s_{0}, t\right)$ is a horizontal Jacobi field along the vertical geodesic $t \mapsto f\left(s_{0}, t\right)$. This field is the basic lift of the vector $\left(\pi \circ \alpha_{0}\right)^{\prime}\left(s_{0}\right)$ restricted to the vertical geodesic. Viewed this way, it is clearly a constant-length Jacobi field. We will refer to these fields as "basic Jacobi fields".

The vector $X \in \mathcal{H}_{p}$ extends naturally to the coordinate vector field $\frac{\partial f}{\partial s}(s, t)=$ $X_{s}(t)$ over the surface (we denote this extension also as $X$ ). Similarly, $V \in \mathcal{V}_{p}$ extends over the surface to the other coordinate vector field $\frac{\partial f}{\partial t}(s, t)=V_{t}(s)$ (we denote this extension also as $V$ ). The $A$-tensor determines the derivative

$$
\nabla_{X} V=\nabla_{V} X=A(X, V),
$$

which is a horizontal vector field along the surface. In particular, at $p$,

$$
V_{0}^{\prime}(0)=X_{0}^{\prime}(0)=A(X, V) \in \mathcal{H}_{p} .
$$

Notice that the roles of $X$ and $V$ are symmetric in the sense that the parameterized surface can be defined in the following two different ways:

$$
f(s, t)=\exp \left(t \cdot V_{0}(s)\right)=\exp \left(s \cdot X_{0}(t)\right) .
$$

In particular, the triple $\{X, V, A(X, V)\}$ is good, which proves Proposition 1.4 


\section{Submersions ARE DEtermined AT A POINT}

It was proven in [1] that a totally geodesic foliation is completely determined by the vertical space and $A$-tensor at a single point:

Proposition 4.1. Suppose that $f_{i}: M \rightarrow B_{i}(i=1,2)$ is a pair of Riemannian submersions with the same complete total space $M$, both with connected totally geodesic fibers. If at a single point $e \in M$ the two vertical spaces agree and the two $A$-tensors agree, then the two Riemannian submersions are the same: $B_{1}=B_{2}$ and $f_{1}=f_{2}$.

Now suppose that $G$ is a compact Lie group with a bi-invariant metric and that $H \subset G$ is a compact Lie subgroup. The left coset space $B=G / H$, or the right coset space $B=H \backslash G$, inherits a unique metric such that the projection $\pi: G \rightarrow B$ becomes a Riemannian submersion, called a (left or right) coset fibration. It is easy to describe the $A$-tensor of $\pi$ at the identity $e \in G$. For $X \in \mathcal{H}_{e}$ and $V \in \mathcal{V}_{e}$, we have

$$
A(X, V)= \pm(1 / 2)[X, V]
$$

(plus for a left coset fibration, and minus for right). The following converse holds:

Corollary 4.2. Let $G$ be a compact Lie group with a bi-invariant metric. Let $\pi: G \rightarrow B$ be a Riemannian submersion with connected totally geodesic fibers. If the A-tensor at the identity $e \in G$ is given by either the plus or the minus version of $A(X, V)= \pm(1 / 2)[X, V]$, then $\pi$ is a coset fibration.

Proof. The vertical space $\mathcal{V}_{e}$ is a subalgebra because for all $U, V \in \mathcal{V}_{e}$ and all $X \in \mathcal{H}_{e}$,

$$
\langle[U, V], X\rangle=\langle[X, U], V\rangle= \pm 2\langle A(X, U), V\rangle=0 .
$$

Let $H$ be the connected Lie subgroup of $G$ with Lie algebra $\mathcal{V}_{e}$. Proposition 4.1 implies that $\pi$ equals a fibration by cosets of $H$.

\section{Bounded Jacobi fields in a compact Lie group}

Let $G$ be a compact Lie group with a bi-invariant metric. In this section, we describe the bounded-length Jacobi fields in $G$.

Let $\mathfrak{g}$ denote the Lie algebra of $G$, and let $X \in \mathfrak{g}$. Let $\gamma(s)=\exp (s X)$. Decompose $\mathfrak{g}$ into eigenspaces of $\operatorname{ad}_{X}^{2}$ :

$$
\mathfrak{g}=\mathfrak{U}_{0} \oplus \sum_{i=1}^{n} \mathfrak{U}_{i}
$$

with corresponding eigenvalues $0=\lambda_{0}^{2}>-\lambda_{1}^{2}>\cdots>-\lambda_{n}^{2}$.

For $V \in \mathfrak{g}$, we let $s \mapsto P_{s} V$ denote its parallel transport along $\gamma$. The Jacobi fields along $\gamma$ are exactly the vector fields of the form

$$
J(s)=P_{s} E_{0}+s \cdot P_{s} F_{0}+\sum_{i=1}^{n} \cos \left(\frac{\lambda_{i} s}{2}\right) \cdot P_{s} E_{i}+\sin \left(\frac{\lambda_{i} s}{2}\right) \cdot P_{s} F_{i},
$$

where $E_{i}, F_{i} \in \mathfrak{U}_{i}$ for each $0 \leq i \leq n$. Equation (5.1) describes the unique Jacobi field along $\gamma$ with the initial data:

$$
J(0)=E_{0}+\sum_{i=1}^{n} E_{i} \quad \text { and } \quad J^{\prime}(0)=F_{0}+\sum_{i=1}^{n} \frac{\lambda_{i}}{2} \cdot F_{i} .
$$


To verify Equation (5.1), it is enough to check that this vector field satisfies the Jacobi equation by using the fact that $R$, the curvature tensor of $G$, is parallel and that $R(X, J) X=-(1 / 4) \operatorname{ad}_{X}^{2}(J)$.

Clearly $J$ has bounded length if and only if $F_{0}=0$. In this case, $J$ has constant length if and only if and for each $1 \leq i \leq n, E_{i}$ and $F_{i}$ are orthogonal and of the same length.

The following provides a useful alternative way to describe bounded Jacobi fields in $G$ :

Lemma 5.1. Any bounded-length Jacobi field in $G$ along $\gamma$ equals the restriction to $\gamma$ of a left-invariant vector field plus a right-invariant vector field.

Proof. For each vector $\mathrm{V}$ in $\mathfrak{U}_{0}$, the left and right extensions of $\mathrm{V}$ along $\gamma$ are the same bounded Jacobi field. For each $\mathrm{V}$ in $\mathfrak{U}_{0}^{\perp}$, the left and right extensions are different bounded Jacobi fields. Taken together, all these fields span a space of bounded Jacobi fields of dimension equal to $\operatorname{dim}\left(\mathfrak{U}_{0}\right)+2 \operatorname{dim}\left(\mathfrak{U}_{0}^{\perp}\right)=\operatorname{dim}(\mathfrak{g})+$ $\operatorname{dim}\left(\mathfrak{U}_{0}^{\perp}\right)$. But this is the dimension of the space of all bounded Jacobi fields along $\gamma$, since each one is determined by its initial value (in $\mathfrak{g}$ ) and its initial derivative (in $\mathfrak{U}_{0}^{\perp}$ ).

In other words, if $J$ is a bounded Jacobi field along $\gamma$, then there exist "left" and "right" vectors $J_{L}, J_{R} \in \mathfrak{g}$ such that $J(s)=d L_{\gamma(s)} J_{L}+d R_{\gamma(s)} J_{R}$. We can always force one of these vectors to lie in $\mathfrak{U}_{0}^{\perp}$. For example, we can force $J_{R}$ to lie in $\mathfrak{U}_{0}^{\perp}$ by subtracting its $\mathfrak{U}_{0}$ component and then adding this component to $J_{L}$. Note that in doing so the Jacobi field does not change. Moreover, $J_{R}$ and $J_{L}$ are uniquely determined once we require $J_{R}$ (or $J_{L}$ ) to lie in $\mathfrak{U}_{0}^{\perp}$.

The pull-back of $J(s)$ to $\mathfrak{g}$ via left multiplication is

$$
\hat{J}(s):=d L_{\gamma(s)^{-1}} J(s)=J_{L}+\operatorname{Ad}_{e^{-s X}}\left(J_{R}\right) .
$$

It is useful to describe this pull-back purely in terms of the Jacobi field's initial data, $V:=J(0)$ and $\mathcal{A}:=J^{\prime}(0)$, rather than $J_{L}$ and $J_{R}$, as follows:

Proposition 5.2. The (bounded or unbounded length) Jacobi field, $J(s)$, in $G$ along $\gamma(s)=\exp (s X)$ with $J(0)=V$ and $J^{\prime}(0)=\mathcal{A}$, pulls back via left multiplication to

$$
\hat{J}(s):=d L_{\gamma(s)^{-1}} J(s)=V+\sum_{n=1}^{\infty} \frac{(-s)^{n}}{n !}\left(\frac{1}{2} \operatorname{ad}_{X}^{n} V-\operatorname{ad}_{X}^{n-1} \mathcal{A}\right) .
$$

Proof. If $J(s)$ is bounded, then we can choose $J_{R}$ and $J_{L}$ as above and notice that $\mathcal{A}=\frac{1}{2}\left[X, J_{L}-J_{R}\right]$, so

$$
J_{L}=\frac{1}{2} V+\operatorname{ad}_{X}^{-1}(\mathcal{A}) \quad \text { and } \quad J_{R}=\frac{1}{2} V-\operatorname{ad}_{X}^{-1}(\mathcal{A}) .
$$

The expression $\operatorname{ad}_{X}^{-1}(\mathcal{A})$ is well-defined because $\mathcal{A}$ is perpendicular to the null-space of $\operatorname{ad}_{X}^{2}$ due to the fact that $J(s)$ is bounded. Substituting these expressions for $J_{L}$ and $J_{R}$ into Equation (5.2) yields

$$
\begin{aligned}
\hat{J}(s) & =\frac{1}{2} V+\operatorname{ad}_{X}^{-1}(\mathcal{A})+\operatorname{Ad}_{e^{-s X}}\left(\frac{1}{2} V-\operatorname{ad}_{X}^{-1}(\mathcal{A})\right) \\
& =V+\sum_{n=1}^{\infty} \frac{(-s)^{n}}{n !}\left(\frac{1}{2} \operatorname{ad}_{X}^{n} V-\operatorname{ad}_{X}^{n-1} \mathcal{A}\right) .
\end{aligned}
$$


If $J(s)$ is unbounded, then the result is obtained by writing $J(s)$ as the sum of a bounded and a linear Jacobi field and by applying the above formula to the bounded part.

\section{Characterization of Good triples in a COMPACT Lie Group}

The goal of this section is to prove Theorem 1.5. Let $G$ be a compact Lie group with bi-invariant metric. Let $\mathfrak{g}$ denote the Lie algebra of $G$. Let $\{X, V, \mathcal{A}\} \subset \mathfrak{g}$. This triple defines a parameterized surface in two ways:

(1) Let $X(t)$ denote the Jacobi field along the geodesic $t \mapsto e^{t V}$ with $X(0)=X$ and $X^{\prime}(0)=\mathcal{A}$, and let $f(s, t)=\exp _{e^{t V}}(s X(t))$.

(2) Let $V(s)$ denote the Jacobi field along the geodesic $s \mapsto e^{s X}$ with $V(0)=V$ and $V^{\prime}(0)=\mathcal{A}$, and let $\tilde{f}(s, t)=\exp _{e^{s X}}(t V(s))$.

Recall that $\{X, V, \mathcal{A}\}$ is called good if and only if $f(s, t)=\tilde{f}(s, t)$ for all $s, t \in \mathbb{R}$. Denote the pull-backs to $\mathfrak{g}$ via left-multiplication as

$$
\hat{X}(t):=d L_{e^{-t V}} X(t) \in \mathfrak{g} \text { and } \hat{V}(s):=d L_{e^{-s X}} V(s) \in \mathfrak{g} .
$$

By Proposition 5.2 (with the roles of $X$ and $V$ interchanged),

$$
\hat{X}^{\prime}(0)=\mathcal{A}+\frac{1}{2} \operatorname{ad}_{X} V
$$

Let $Y(s, t) \in \mathfrak{g}$ denote the pull-back to $\mathfrak{g}$ via left-multiplication of the $\frac{\partial}{\partial t}$ coordinate vector field of the parameterizes surface $f$; that is, $Y(s, t):=d L_{f(s, t)^{-1}} \frac{\partial f}{\partial t}(s, t)$. Using Proposition 5.2 and Equation (6.1), we have

$$
\begin{aligned}
Y(s, 0)=\hat{V}(s) & =V+\sum_{n=1}^{\infty} \frac{(-s)^{n}}{n !}\left(\frac{1}{2} \operatorname{ad}_{X}^{n} V-\operatorname{ad}_{X}^{n-1} \mathcal{A}\right) \\
& =V+\sum_{n=1}^{\infty} \frac{(-s)^{n}}{n !}\left(\operatorname{ad}_{X}^{n} V-\operatorname{ad}_{X}^{n-1}\left(\hat{X}^{\prime}(0)\right)\right) .
\end{aligned}
$$

It is straightforward to generalize this to the case where " 0 " is replaced by an arbitrary $t \in \mathbb{R}$ :

$$
Y(s, t)=V+\sum_{n=1}^{\infty} \frac{(-s)^{n}}{n !}\left(\operatorname{ad}_{\hat{X}(t)}^{n} V-\operatorname{ad}_{\hat{X}(t)}^{n-1}\left(\hat{X}^{\prime}(t)\right)\right) .
$$

Now assume that $\{X, V, \mathcal{A}\}$ is a good triple so that for each fixed $s$, the curve $t \mapsto f(s, t)$ is a geodesic. In a compact Lie group with a bi-invariant metric, a geodesic's velocity field is obtained by left-translating its initial velocity vector; that is, $\frac{\partial}{\partial t} f(s, t)=d L_{f(s, t) \cdot f(s, 0)^{-1}}\left(\frac{\partial}{\partial t} f(s, 0)\right)$. Therefore,

$$
Y(s, t)=d L_{e^{-s X}}(V(s))=\hat{V}(s)=Y(s, 0) .
$$

Thus Equation (6.2) does not depend on $t$, resulting in:

Condition 6.1. For each $n \geq 1$, the expression $\operatorname{ad}_{\hat{X}(t)}^{n} V-\operatorname{ad}_{\hat{X}(t)}^{n-1}\left(\hat{X}^{\prime}(t)\right)$ is independent of $t$.

In fact, Condition 6.1 is equivalent to the condition that $\{X, V, \mathcal{A}\}$ is good. To see this, observe that Condition 6.1 implies that for each fixed $s$, the curve $t \mapsto f(s, t)$ is a geodesic. By definition, $f(s, 0)=\tilde{f}(s, 0)$ and $\frac{\partial f}{\partial t}(s, 0)=\frac{\partial \tilde{f}}{\partial t}(s, 0)$, so the geodesics 
$t \mapsto \tilde{f}(s, t)$ and $t \mapsto f(s, t)$ must agree (as they are geodesics with the same initial position and velocity), which implies that $\tilde{f}=f$.

Next we claim that Condition 6.1 is equivalent to the following:

Condition 6.2. For each $m \geq 0$, the expression $\operatorname{ad}_{\hat{X}(t)}^{m}\left(\frac{1}{2} \operatorname{ad}_{X} V-\mathcal{A}\right)$ is independent of $t$.

To demonstrate this, first re-write the expression in Condition 6.1 as

$$
\operatorname{ad}_{\hat{X}(t)}^{n} V-\operatorname{ad}_{\hat{X}(t)}^{n-1}\left(\hat{X}^{\prime}(t)\right)=\operatorname{ad}_{\hat{X}(t)}^{n-1}\left(\operatorname{ad}_{\hat{X}(t)} V-\hat{X}^{\prime}(t)\right),
$$

so it remains to simplify the above inner expression as

$$
\operatorname{ad}_{\hat{X}(t)} V-\hat{X}^{\prime}(t)=-\operatorname{ad}_{V} \hat{X}(t)-\hat{X}^{\prime}(t)=\frac{1}{2} \operatorname{ad}_{X} V-\mathcal{A} .
$$

To justify the second equality in Equation (6.3), we use Proposition 5.2 (with the roles $X$ and $V$ interchanged) to express $\hat{X}(t)$ as

$$
\hat{X}(t)=X+\sum_{n=1}^{\infty} \frac{(-t)^{n}}{n !}\left(\frac{1}{2} \operatorname{ad}_{V}^{n} X-\operatorname{ad}_{V}^{n-1} \mathcal{A}\right),
$$

SO

$$
\begin{aligned}
\operatorname{ad}_{V} \hat{X}(t)+\hat{X}^{\prime}(t)= & \operatorname{ad}_{V} X+\sum_{n=1}^{\infty} \frac{(-t)^{n}}{n !}\left(\frac{1}{2} \operatorname{ad}_{V}^{n+1} X-\operatorname{ad}_{V}^{n} \mathcal{A}\right) \\
& +\left(\mathcal{A}-\frac{1}{2} \operatorname{ad}_{V} X\right)+\sum_{n=1}^{\infty}-\frac{(-t)^{n}}{n !}\left(\frac{1}{2} \operatorname{ad}_{V}^{n+1} X-\operatorname{ad}_{V}^{n} \mathcal{A}\right) \\
= & \mathcal{A}+\frac{1}{2} \operatorname{ad}_{V} X=\mathcal{A}-\frac{1}{2} \operatorname{ad}_{X} V .
\end{aligned}
$$

This verifies that Condition 6.2 is equivalent to Condition 6.1 and is therefore equivalent to the condition that $\{X, V, \mathcal{A}\}$ is a good triple.

It remains to show that Condition 6.2 is equivalent to the condition in Theorem 1.5. Condition 6.2 says that for each $n \geq 0$, the expression $\operatorname{ad}_{\hat{X}(t)}^{n}(B)$ is independent of $t$. Using Equation (6.4), this implies that

$$
\begin{aligned}
\operatorname{ad}_{X}^{n+1} B & =\operatorname{ad}_{\hat{X}(t)}\left(\operatorname{ad}_{\hat{X}(t)}^{n} B\right)=\operatorname{ad}_{\hat{X}(t)}\left(\operatorname{ad}_{X}^{n} B\right) \\
& =\operatorname{ad}_{X}^{n+1} B+\left[\sum_{m=0}^{\infty} \frac{(-t)^{m+1}}{(m+1) !} \operatorname{ad}_{V}^{m} \bar{B}, \operatorname{ad}_{X}^{n} B\right],
\end{aligned}
$$

so $\left[\operatorname{ad}_{V}^{m} \bar{B}, \operatorname{ad}_{X}^{n} B\right]=0$ for all $n, m \geq 0$. On the other hand, if $\left[\operatorname{ad}_{V}^{m} \bar{B}, \operatorname{ad}_{X}^{n} B\right]=0$ for all $n, m \geq 0$, then the equality of the second and fourth terms of Equation 6.5 gives $\operatorname{ad}_{\hat{X}(t)}^{n+1}(B)=\operatorname{ad}_{X}^{n+1} B$ for all $n \geq 0$, which is exactly Condition 6.2. This ends the proof of Theorem 1.5

\section{Alternative Characterization of GOod triples}

Theorem 1.5 states that the triple $\{X, V, \mathcal{A}\} \subset \mathfrak{g}$ is good if and only if $\left[\operatorname{ad}_{X}^{n} B, \operatorname{ad}_{X}^{m} \bar{B}\right]=0$ for all $n, m \geq 0$, where $B=\frac{1}{2}[X, V]-\mathcal{A}$ and $\bar{B}=\frac{1}{2}[V, X]-\mathcal{A}$. This condition is symmetric in $X$ and $V$, as expected. However, for Riemannian submersions with totally geodesic fibers, holonomy Jacobi fields differ from basic 
Jacobi fields, so it is useful to break the symmetry by focusing on only one type of Jacobi field. Therefore, we will now derive conditions under which a single bounded Jacobi field comes from a good triple. These new conditions will be used in Section9 to construct examples of good triples which do not arise from submersions.

Let $X \in \mathfrak{g}$, and let $V(s)$ be a bounded Jacobi field along the geodesic $s \mapsto e^{s X}$. Choose $V_{L}, V_{R} \in \mathfrak{g}$, with $V_{R}$ orthogonal to the null-space of $\operatorname{ad}_{X}^{2}$, such that

$$
\hat{V}(s):=d L_{e^{-s X}} V(s)=V_{L}+\operatorname{Ad}_{e^{-s X}} V_{R},
$$

as in Equation (5.2). Define $V:=V(0)=V_{L}+V_{R}$ and $\mathcal{A}:=V^{\prime}(0)=\frac{1}{2}\left[X, V_{L}-V_{R}\right]$. We seek conditions in terms of $\left\{X, V_{L}, V_{R}\right\}$ under which the triple $\{X, V, \mathcal{A}\}$ is good.

Corollary 7.1. Let $G$ be a compact Lie group with a bi-invariant metric. Let $X, V_{L}, V_{R} \in \mathfrak{g}$. Assume that $V_{R}$ is orthogonal to the null-space of $a d_{X}^{2}$. Then the triple

$$
\left\{X, V_{L}+V_{R}, \frac{1}{2}\left[X, V_{L}-V_{R}\right]\right\}
$$

is good if and only the following two conditions are satisfied:

(1) $\left[\operatorname{Ad}_{e^{-t X}} V_{R}, \operatorname{ad}_{X} V_{L}\right]=0$ for all $t \in \mathbb{R}$, and

(2) $\left[\operatorname{ad}_{V_{L}}^{m} X,\left[\operatorname{Ad}_{e^{-t X}} V_{R}, V_{L}\right]\right]=0$ for all $t \in \mathbb{R}$ and all $m \geq 1$.

The remainder of this section is devoted to the proof of Corollary 7.1. Our strategy is to prove that the condition of Theorem 1.5 (above) is equivalent to Condition 7.2 (below), which in turn is equivalent to Condition 7.3 (below), which in turn is equivalent to the conditions of Corollary 7.1 .

Condition 7.2. For each $n \geq 0, \operatorname{ad}_{\hat{V}(s)}^{n}(\bar{B})$ is constant in $s$.

Condition 7.3. $\left[\operatorname{Ad}_{e^{-s X}} V_{R}, \operatorname{ad}_{V_{L}}^{n} X\right]=0$ for all $s \in \mathbb{R}$ and all $n \geq 1$.

To begin, let us consider a root-space decomposition,

$$
\mathfrak{g}=\tau \oplus_{i \in I} \mathfrak{l}_{i}
$$

where $\tau$ is a maximal abelian subalgebra containing $X$ and $I$ is the index set for the two-dimensional root spaces $\mathfrak{l}_{i}$. For $W \in \mathfrak{g}$, let $W^{i}$ denote the orthogonal projection of $W$ onto $\mathfrak{l}_{i}$ and let $W^{0}$ denote the projection onto $\tau$.

Lemma 7.4. Let $W, U \in \mathfrak{g}$. Assume $W$ is orthogonal to the null-space of $a d_{X}^{2}$. If $\left[A d_{e^{-s X}} W, U\right]$ is constant in $s$, then it vanishes.

Proof. Assume that

$$
\begin{aligned}
{\left[\operatorname{Ad}_{e^{-s x}} W, U\right] } & =\left[\sum_{i \in I} \operatorname{Ad}_{e^{-s x}} W^{i}, U^{0}+\sum_{j \in I} U^{j}\right] \\
& =\sum_{i \in I}\left[\operatorname{Ad}_{e^{-t x}} W^{i}, U^{0}\right]+\sum_{i, j \in I}\left[\operatorname{Ad}_{e^{-t X}} W^{i}, U^{j}\right]
\end{aligned}
$$

is constant in $s$. The $\tau$-component of this expression comes from the diagonal $i=j$ terms of the double sum. Each such diagonal term has the form $C_{i} \cos \left(\lambda_{i}+a_{i} t\right) r_{i}$ for some $C_{i}, \lambda_{i}, a_{i} \in \mathbb{R}$, where $r_{i} \in \tau$ is the associated dual root. The only way that several terms of this form could sum to a constant function is if they sum to zero.

Similarly, every term of the single-sum and every non-diagonal term of the double-sum above equals a circle in a single root space. That is, it has the form 
$C \cos (\lambda+a t) E+C \sin (\lambda+a t) F$, where $\{E, F\}$ is an orthonormal basis of one of the $\mathfrak{l}_{i}$ 's. The only way that several such circles could cancel so as to sum to a constant function is if they sum to zero.

Proof of Corollary 7.1. Notice that $B=\left[X, V_{R}\right]$ and $\bar{B}=-\left[X, V_{L}\right]$, so in this setting Condition 6.2 from the previous section (with the roles of $X$ and $V$ reversed) says $\left\{X, V_{L}+V_{R}, \frac{1}{2}\left[X, V_{L}-V_{R}\right]\right\}$ is good if and only if Condition 7.2 is satisfied.

Next we prove that Condition 7.2 is equivalent to Condition 7.3 First assume that Condition 7.2 holds. The $n=1$ case of this condition says that the following is constant in $s$ :

$$
[\hat{V}(s), \bar{B}]=\left[V_{L}+\operatorname{Ad}_{e^{-s X}} V_{R},-\left[X, V_{L}\right]\right]=\operatorname{ad}_{V_{L}}^{2} X-\left[\operatorname{Ad}_{e^{-s X}} V_{R},\left[X, V_{L}\right]\right] .
$$

Lemma 7.4 implies that the second term of this last expression vanishes, so

$$
\left[\operatorname{Ad}_{e^{-s X}} V_{R},\left[X, V_{L}\right]\right]=0 \text { and }[\hat{V}(s), \bar{B}]=\operatorname{ad}_{V_{L}}^{2} X \text {. }
$$

Similarly, the $n=2$ case of Condition 7.2 gives that

$$
[\hat{V}(s),[\hat{V}(s), \bar{B}]]=\left[V_{L}+\operatorname{Ad}_{e^{-s x}} V_{R}, \operatorname{ad}_{V_{L}}^{2} X\right]=\operatorname{ad}_{V_{L}}^{3} X+\left[\operatorname{Ad}_{e^{-s X}} V_{R}, \operatorname{ad}_{V_{L}}^{2} X\right]
$$

is constant in $s$, which implies that

$$
\left[\operatorname{Ad}_{e^{-s X}} V_{R}, \operatorname{ad}_{V_{L}}^{2} X\right]=0 \text { and } \operatorname{ad}_{\hat{V}(s)}^{2} \bar{B}=\operatorname{ad}_{V_{L}}^{3} X .
$$

Continue by induction to get that for all $n \geq 1$,

$$
\left[\operatorname{Ad}_{e^{-s X}} V_{R}, \operatorname{ad}_{V_{L}}^{n} X\right]=0 \text { and } \operatorname{ad}_{\hat{V}(s)}^{n} \bar{B}=\operatorname{ad}_{V_{L}}^{n+1} X .
$$

In particular, Condition 7.3 is true. In fact, the above work shows that Condition 7.3 is equivalent to Condition 7.2, so it remains to show that Condition 7.3 is equivalent to the two conditions of Corollary 7.1. Indeed, the $n=1$ case of Condition 7.3 coincides with condition (1) of Corollary 7.1. Moreover, by the Jacobi identity we have

$$
\begin{aligned}
{\left[\operatorname{ad}_{V_{L}}^{m} X,\left[\operatorname{Ad}_{e^{-s X}} V_{R}, V_{L}\right]\right] } & =-\left[\operatorname{Ad}_{e^{-s X}} V_{R},\left[V_{L}, \operatorname{ad}_{V_{L}}^{m} X\right]\right]-\left[V_{L},\left[\operatorname{ad}_{V_{L}}^{m} X, \operatorname{Ad}_{e^{-s X} V_{R}}\right]\right] \\
& =-\left[\operatorname{Ad}_{e^{-s X}} V_{R}, \operatorname{ad}_{V_{L}}^{m+1} X\right]+\operatorname{ad}_{V_{L}}\left[\operatorname{Ad}_{e^{-s X}} V_{R}, \operatorname{ad}_{V_{L}}^{m} X\right]
\end{aligned}
$$

which completes the proof.

\section{Constant length Jacobi fields and good triples}

In this section, we prove that good triples induce constant-length Jacobi fields, provided they induce bounded-length Jacobi fields, and we also prove a related finiteness result, which requires the following definition:

Definition 8.1. Let $G$ be a compact Lie group and let $\mathfrak{g}$ denote its Lie algebra. The vector $X \in \mathfrak{g}$ is called "weakly regular" if for any nonzero eigenvalue of $\operatorname{ad}_{X}^{2}$, the collection of roots associated to the root spaces which comprise the corresponding eigenspace is linearly independent.

Typically, $X \in \mathfrak{g}$ is called "regular" if it lies in a unique maximal abelian subalgebra of $\mathfrak{g}$. In this case, the nullspace of $\operatorname{ad}_{X}^{2}$ equals this maximal abelian subalgebra, and each eigenspaces of $\operatorname{ad}_{X}^{2}$ corresponding to a nonzero eigenvalue equals a root space or possibly a direct sum of multiple root spaces. If each eigenspace equals a single root space, then $X$ is called "strongly regular". In our definition of "weakly regular" above, we are allowing the null-space of $\operatorname{ad}_{X}^{2}$ to be larger than a maximal abelian subalgebra. By choosing an arbitrary maximal abelian subalgebra inside 
this null-space, we can consider a root space decomposition with respect to the chosen subalgebra. Each eigenspace of $\operatorname{ad}_{X}^{2}$ corresponding to a nonzero eigenvalue will equal a single root space or a direct sum of multiple root spaces. Our definition allows it to be a direct sum of multiple root spaces, as long as the corresponding collection of multiple roots is linearly independent. The conditions "regular", "strongly regular" and "weakly regular" are clearly generic conditions, meaning that each condition is satisfied by an open and dense collection of vectors in $\mathfrak{g}$.

Proposition 8.2. Let $G$ be a compact Lie group with a bi-invariant metric. Let $\mathfrak{g}$ denote its Lie algebra. Let $\{X, V, \mathcal{A}\} \subset \mathfrak{g}$ be a good triple. Assume that the Jacobi field $V(s)$ (defined as in Definition 1.3) has bounded length.

(1) The Jacobi field $V(s)$ has constant-length.

(2) If $X$ is weakly regular, then $\mathcal{A}$ projects onto each root space (with respect to a maximal abelian subalgebra containing $X$ ) as plus or minus half the bracket of $X$ with the projection of $V$ onto this root space. In particular, there are only finitely many possibilities for $\mathcal{A}$ (corresponding to the possible sign choices, not all of which necessarily yield good triples).

If $X$ has the stronger regularity property that each eigenspace is comprised of a single root space, then notice that the conclusion of part (2) follows from part (1) together with our previous classification of constant length Jacobi fields.

Proof. As in Lemma [5.1] choose $V_{L}, V_{R} \in \mathfrak{g}$ such that $V=V_{L}+V_{R}$ and $\mathcal{A}=$ $\frac{1}{2}\left[X, V_{L}-V_{R}\right]$ and $V_{R}$ is orthogonal to the null-space of $\operatorname{ad}_{X}^{2}$. To prove part (1) of the proposition, it suffices to verify that the norm of $\hat{V}(s):=V_{L}+\operatorname{Ad}_{e^{-s X}} V_{R}$ is independent of $s$, since this is the left pull-back of one of the Jacobi fields determined by the good triple.

As before, consider a root-space decomposition, $\mathfrak{g}=\tau \oplus_{i \in I} \mathfrak{l}_{i}$, where $\tau$ is a maximal abelian subalgebra containing $X$ and $I$ is the index set for the 2-dimensional root spaces $\mathfrak{l}_{i}$. For $W \in \mathfrak{g}$, let $W^{i}$ denote the orthogonal projection of $W$ onto $\mathfrak{l}_{i}$ and let $W^{0}$ denote the projection onto $\tau$. Part (2) of the proposition is equivalent to the following: if $X$ is weakly regular, then for each index $i$, either $V_{L}^{i}=0$ or $V_{R}^{i}=0$.

Each eigenspace of $\operatorname{ad}_{X}^{2}$ equals a sum of a subset of the root spaces. Choose a fixed such eigenspace, $\Lambda=\bigoplus_{i \in I^{\prime}} \mathfrak{l}_{i}$, where $I^{\prime} \subset I$ is the corresponding subset of indices. For notational convenience, we assume without loss of generality that the corresponding eigvalue equals 1 . Condition 7.3 says that for all $n \geq 1$ and all $s \in \mathbb{R}$, we have

$$
\sum_{i \in I}\left[\operatorname{Ad}_{e^{-s X}} V_{R}^{i}, \operatorname{ad}_{V_{L}}^{n} X\right]=0 .
$$

Two nonvanishing terms of this sum have the same period if and only if the corresponding two root spaces are grouped into the same eigenspace of $\operatorname{ad}_{X}^{2}$. Terms with different periods cannot cancel each other, so the sum over $I^{\prime}$ alone vanishes:

$$
\sum_{i \in I^{\prime}}\left[\operatorname{Ad}_{e^{-s X}} V_{R}^{i}, \operatorname{ad}_{V_{L}}^{n} X\right]=0 .
$$

Notice that $\operatorname{ad}_{X}$ induces an orientation on each $\mathfrak{l}_{i} \subset \Lambda$. For each $i \in I^{\prime}$, let $\theta_{i} \in[0,2 \pi)$ denote the oriented angle from $V_{L}^{i}$ to $V_{R}^{i}$; that is, the smallest value of $\theta$ such that $\operatorname{Ad}_{e^{\theta X}} V_{L}^{i}$ is a positive scalar multiple of $V_{R}^{i}$. Also denote $A_{i}:=\left|V_{L}^{i}\right| \cdot\left|V_{R}^{i}\right|$. Finally, let $\hat{r}_{i} \in \tau$ denote the corresponding dual-root; that is, $\hat{r}_{i}=\left[W, \operatorname{ad}_{X} W\right]$ for 
any unit-length $W \in \mathfrak{l}_{i}$. It is useful to re-define $I^{\prime}$ by removing all indices $i$ for which $A_{i}=0$. This way, the angle $\theta_{i}$ is well-defined for each $i \in I^{\prime}$.

The $n=1$ case of Equation (8.1) says that

$$
\sum_{i \in I^{\prime}}\left[\operatorname{Ad}_{e^{-s X}} V_{R}^{i}, \operatorname{ad}_{X} V_{L}\right]=0 .
$$

In particular, the projection of this sum of brackets onto $\tau$ vanishes, so

$$
0=\sum_{i \in I^{\prime}}\left[\operatorname{Ad}_{e^{-s X}} V_{R}^{i}, \operatorname{ad}_{X} V_{L}^{i}\right]=\sum_{i \in I^{\prime}} A_{i} \cos \left(\theta_{i}-s\right) \cdot \hat{r}_{i} .
$$

If $X$ is weakly regular, then the $\hat{r}_{i}$ 's are linearly independent, so each $A_{i}$ must vanish, which proves part (2) of the proposition.

Part (1) of the proposition is proven by taking the inner product with $X$ :

$$
0=\sum_{i \in I^{\prime}} A_{i} \cos \left(\theta_{i}-s\right)\left\langle\hat{r}_{i}, X\right\rangle=\sum_{i \in I^{\prime}} A_{i} \cos \left(\theta_{i}-s\right) .
$$

The condition that $\hat{V}(s)=V_{L}+\operatorname{Ad}_{e^{-s x}} V_{R}$ has constant length means that the projection of $\hat{V}(s)$ onto each such eigenspace $\Lambda$ has constant length, which is equivalent to the following being constant in $s$ :

$$
f(s)=\left\langle V_{L}^{\Lambda}, \operatorname{Ad}_{e^{-s X}} V_{R}^{\Lambda}\right\rangle=\sum_{i \in I^{\prime}}\left\langle V_{L}^{i}, \operatorname{Ad}_{e^{-s X}} V_{R}^{i}\right\rangle=\sum_{i \in I^{\prime}} A_{i} \cos \left(\theta_{i}-s\right) .
$$

Comparing this to Equation (8.3) verifies that $\hat{V}(s)$ has constant length.

\section{Examples of good triples in a COMPaCt Lie GRoup}

In this section, we present two different methods for constructing a good triple in a simple compact Lie group which does not come from a coset fibration. Our first method produces a triple $\{X, V, \mathcal{A}\}$ which does satisfy the conclusion of Proposition $8.2(2)$. Thus, once $X$ and $V$ are chosen, there are only finitely many "sign" choices for $\mathcal{A}$. We demonstrate that a mixed sign choice can still produce a good triple. Our second method uses a vector $X$ which is not weakly regular and is contrived so that the conclusion of Proposition 8.2(2) does NOT hold.

The idea of our first method is to construct one-dimensional biquotient submersions which have totally geodesic fibers along certain horizontal geodesics, so that the discussion in Section 3 applies along these horizontal geodesics.

Let $G$ be a simple compact Lie group with a bi-invariant metric, let $\mathfrak{g}$ denote its Lie algebra, and let $X \in \mathfrak{g}$. With respect to a maximal abelian subalgebra of $\mathfrak{g}$ containing $X$, suppose that there are two roots, $\alpha$ and $\beta$, such that $\alpha \pm \beta$ is not a root and such that $\alpha(X) \neq 0$ and $\beta(X) \neq 0$. In this case, choose vectors $V_{\alpha}, V_{\beta} \in \mathfrak{g}$ from the corresponding two root spaces. Notice that

$$
\left[\operatorname{Ad}_{e^{s X}} V_{\alpha}, V_{\beta}\right]=0 \text { for all } s \in \mathbb{R} .
$$

This pair of vectors determines an $\mathbb{R}$-action on $G$, defined so that for $g \in G$ and $t \in \mathbb{R}, t \star g:=\left(e^{t V_{\alpha}}\right) \cdot g \cdot\left(e^{-t V_{\beta}}\right)$. The vertical space at $\mathfrak{g}$ of the Riemannian submersion $\pi: G \rightarrow G / \mathbb{R}$ is spanned by $V:=V_{\alpha}-V_{\beta}$. Notice that $\gamma(s):=e^{s X}$ is a horizontal geodesic because it begins (and therefore remains) perpendicular to the vertical space. We claim that each fiber of $\pi$ which intersects $\gamma$ is totally geodesic. 
Using Equation (9.1), for fixed $s \in \mathbb{R}$, the image of the fiber through $\gamma(s)$ under the left-translation map $L_{\gamma(s)^{-1}}$ equals the path

$$
t \mapsto e^{-s X} e^{t V_{\alpha}} e^{s X} e^{-t V_{\beta}}=e^{\left(\operatorname{Ad}_{e^{-s X}}\left(t V_{\alpha}\right)\right)} \cdot e^{-t V_{\beta}}=e^{t\left(\operatorname{Ad}_{-s X} V_{\alpha}-V_{\beta}\right)},
$$

which is the geodesic in the direction of $\operatorname{Ad}_{-s X} V_{\alpha}-V_{\beta}$. Since the fibers along $\gamma$ are totally geodesic, the discussion in Section 3 establishes that $\{X, V, \mathcal{A}\}$ is a good triple, where $\mathcal{A}:=A(X, V)=-\frac{1}{2}\left[X, V_{\alpha}+V_{\beta}\right]$. Alternately, it is straightforward to verify that this triple is good directly from Theorem 1.5. Since $\alpha(X) \neq 0$ and $\beta(X) \neq 0$, notice that $A(X, V) \neq \pm \frac{1}{2}[X, V]$, which distinguishes this biquotient fibration from coset fibrations.

The idea of our second construction method is to use the following, which follows immediately from Corollary 7.1 ,

Proposition 9.1. If $\left\{X, V_{L}, V_{R}\right\} \subset \mathfrak{g}$ satisfy the following properties:

(1) $V_{L}$ and $V_{R}$ lie in a single eigenspace of $a d_{X}^{2}$,

(2) $\left[\operatorname{Ad}_{e^{-t X}} V_{R}, V_{L}\right]=0$ for all $t \in \mathbb{R}$, then $\left\{X, V_{L}+V_{R}, \frac{1}{2}\left[X, V_{L}-V_{R}\right]\right\}$ is a good triple.

To describe our second example, let

$$
G=S U(4) \text { and } X=\operatorname{diag}(-\mathbf{i}, 0, \mathbf{i}, 0) \in \mathfrak{g}=s u(4) .
$$

Notice that +1 is an eigenvalue of $\operatorname{ad}_{X}^{2}$ whose corresponding eigenspace is comprised of four root spaces, commonly denoted as $\mathfrak{l}_{12}, \mathfrak{l}_{23}, \mathfrak{l}_{34}, \mathfrak{l}_{14}$. Let $\left\{E_{i j}, F_{i j}\right\} \subset \mathfrak{g}$ denote the standard basis of $\mathfrak{l}_{i j}$; that is, $E_{i j}$ has 1 in position $(i, j)$ and -1 in position $(j, i)$, while $F_{i j}$ has $\mathbf{i}$ in positions $(i, j)$ and $(j, i)$. Define

$$
V_{L}=E_{12}+E_{23}+E_{34}+E_{14} \text { and } V_{R}=F_{12}+F_{23}+F_{34}-F_{14} \text {. }
$$

We claim that $\left[\operatorname{Ad}_{e^{-t x}} V_{R}, V_{L}\right]=0$ for all $t \in \mathbb{R}$, and therefore that the triple $\left\{X, V_{L}+V_{R}, \frac{1}{2}\left[X, V_{L}-V_{R}\right]\right\}$ is good. It is sufficient to verify this at $t=0$ and $t=\pi / 2$, which is a straightforward calculation.

In this example, there are root spaces onto which $V_{L}$ and $V_{R}$ both have nonzero projections. In particular, this example does not satisfy the conclusion of part (2) of Proposition 8.2

\section{GoOd triples in LOCAlly SyMmetric SPACES}

In this section, we prove Proposition [1.6, which provides a necessary condition for a triple to be good in any locally symmetric space.

Proof of Proposition 1.6. Consider the surface $f(s, t)=\exp (t \cdot V(s))=\exp (s \cdot X(t))$ induced by a good triple $\{X, V, \mathcal{A}\} \subset T_{p} M$ as in Definition 1.3 and introduce the following notation: $X_{s, t}:=f_{*(s, t)} \frac{\partial}{\partial s}=\frac{\partial f}{\partial s}(s, t), V_{s, t}:=f_{*(s, t)} \frac{\partial}{\partial t}=\frac{\partial f}{\partial t}(s, t)$, and $A_{s, t}:=\nabla_{\frac{\partial}{\partial t}} X_{s, t}$. (Notice that $X_{0, t}=X(t)$ and $V_{s, 0}=V(s)$.)

The formulas below are well known to hold for general parametrized surfaces:

$$
\begin{gathered}
\nabla_{\frac{\partial}{\partial t}} X_{s, t}=\nabla_{\frac{\partial}{\partial s}} V_{s, t}, \\
\nabla_{\frac{\partial}{\partial s}} \nabla_{\frac{\partial}{\partial t}} Y_{s, t}-\nabla_{\frac{\partial}{\partial t}} \nabla_{\frac{\partial}{\partial s}} Y_{s, t}=R\left(X_{s, t}, V_{s, t}\right) Y_{s, t},
\end{gathered}
$$

for any vector field $Y$ along $f$.

Using (10.2) with $Y_{s, t}=X_{s, t}$, we obtain

$$
\nabla_{\frac{\partial}{\partial s}} \nabla_{\frac{\partial}{\partial t}} X_{s, t}-\nabla_{\frac{\partial}{\partial t}} \nabla_{\frac{\partial}{\partial s}} X_{s, t}=R\left(X_{s, t}, V_{s, t}\right) X_{s, t}
$$


but $s \rightarrow f(s, t)$ is a geodesic, so $\nabla_{\frac{\partial}{\partial s}} X_{s, t}=\nabla_{\frac{\partial}{\partial s}} \frac{\partial f}{\partial s}=0$. This way,

$$
\nabla_{\frac{\partial}{\partial s}} \nabla_{\frac{\partial}{\partial t}} X_{s, t}=R\left(X_{s, t}, V_{s, t}\right) X_{s, t}
$$

Similarly,

$$
-\nabla_{\frac{\partial}{\partial t}} \nabla_{\frac{\partial}{\partial s}} V_{s, t}=R\left(X_{s, t}, V_{s, t}\right) V_{s, t},
$$

by using (10.2) with $Y_{s, t}=V_{s, t}$.

Applying $\nabla_{\frac{\partial}{\partial t}}$ in (10.3), $\nabla_{\frac{\partial}{\partial s}}$ in (10.4), and adding the two relations yields $(10.5)$

$\nabla_{\frac{\partial}{\partial t}} \nabla_{\frac{\partial}{\partial s}} \nabla_{\frac{\partial}{\partial t}} X_{s, t}-\nabla_{\frac{\partial}{\partial s}} \nabla_{\frac{\partial}{\partial t}} \nabla_{\frac{\partial}{\partial s}} V_{s, t}=\nabla_{\frac{\partial}{\partial t}} R\left(X_{s, t}, V_{s, t}\right) X_{s, t}+\nabla_{\frac{\partial}{\partial s}} R\left(X_{s, t}, V_{s, t}\right) V_{s, t}$.

Observe that, since the manifold is locally symmetric,

$$
\begin{aligned}
\nabla_{\frac{\partial}{\partial t}} R\left(X_{s, t}, V_{s, t}\right) X_{s, t}= & R\left(\nabla_{\frac{\partial}{\partial t}} X_{s, t}, V_{s, t}\right) X_{s, t} \\
& +R\left(X_{s, t}, \nabla_{\frac{\partial}{\partial t}} V_{s, t}\right) X_{s, t}+R\left(X_{s, t}, V_{s, t}\right) \nabla_{\frac{\partial}{\partial t}} X_{s, t} .
\end{aligned}
$$

As the second term on the right side of the equality above is zero due to the fact that $t \rightarrow f(s, t)$ is a geodesic, we have

$$
\nabla_{\frac{\partial}{\partial t}} R\left(X_{s, t}, V_{s, t}\right) X_{s, t}=R\left(\nabla_{\frac{\partial}{\partial t}} X_{s, t}, V_{s, t}\right) X_{s, t}+R\left(X_{s, t}, V_{s, t}\right) A_{s, t} .
$$

Similarly, based on the same type of computations as above and relation (10.1),

$$
\nabla_{\frac{\partial}{\partial s}} R\left(X_{s, t}, V_{s, t}\right) V_{s, t}=R\left(X_{s, t}, \nabla_{\frac{\partial}{\partial s}} V_{s, t}\right) X_{s, t}+R\left(X_{s, t}, V_{s, t}\right) A_{s, t} .
$$

By adding relations (10.6) and (10.7), it follows that the term on the right side of relation (10.5) is

$$
\begin{aligned}
& \nabla_{\frac{\partial}{\partial t}} R\left(X_{s, t}, V_{s, t}\right) X_{s, t}+\nabla_{\frac{\partial}{\partial s}} R\left(X_{s, t}, V_{s, t}\right) V_{s, t} \\
& \quad=R\left(X_{s, t}, A_{s, t}\right) V_{s, t}+R\left(A_{s, t}, V_{s, t}\right) X_{s, t}+2 R\left(X_{s, t}, V_{s, t}\right) A_{s, t} .
\end{aligned}
$$

On the other hand, by using relation (10.2) with $Y_{s, t}=\nabla_{\frac{\partial}{\partial t}} X_{s, t}=\nabla_{\frac{\partial}{\partial s}} V_{s, t}=A_{s, t}$, the term on the left side of relation (10.5) can be written as

$$
\nabla_{\frac{\partial}{\partial t}} \nabla_{\frac{\partial}{\partial s}} \nabla_{\frac{\partial}{\partial t}} X_{s, t}-\nabla_{\frac{\partial}{\partial s}} \nabla_{\frac{\partial}{\partial t}} \nabla_{\frac{\partial}{\partial s}} V_{s, t}=R\left(X_{s, t}, V_{s, t}\right) A_{s, t} .
$$

Based on the two formulas above, relation (10.5) becomes

$$
R\left(X_{s, t}, A_{s, t}\right) V_{s, t}+R\left(A_{s, t}, V_{s, t}\right) X_{s, t}+R\left(X_{s, t}, V_{s, t}\right) A_{s, t}=0 .
$$

But, by the first Bianchi identity, we have

$$
R\left(X_{s, t}, V_{s, t}\right) A_{s, t}+R\left(V_{s, t}, A_{s, t}\right) X_{s, t}+R\left(A_{s, t}, X_{s, t}\right) V_{s, t}=0 .
$$

So,

$$
R\left(A_{s, t}, V_{s, t}\right) X_{s, t}+R\left(X_{s, t}, A_{s, t}\right) V_{s, t}=R\left(X_{s, t}, V_{s, t}\right) A_{s, t} .
$$

Combining relations (10.8) and (10.9), we obtain

$$
R\left(X_{s, t}, V_{s, t}\right) A_{s, t}=0 .
$$

When $t=s=0$, relation (10.10) becomes $R(X, V) \mathcal{A}=0$, as claimed. 


\section{ACKNOWLEDGEMENTS}

It is our pleasure to thank Craig Sutton for many helpful discussions and the referee for valuable suggestions. We also wish to thank Dartmouth College and AIM for providing hospitality. Part of this work was completed at the AIM workshop on nonnnegative curvature in September 2007.

\section{REFERENCES}

1. R. Escobales, Riemannian submersions with totally geodesic fibers, J. Diff. Geom. 10 (1975), 253-276. MR0370423 (51:6650)

2. R. Escobales, Riemannian submersions from complex projective space, J. Diff. Geom. 13 (1978), 93-107. MR520604 (80k:53106)

3. L. Florit, Doubly ruled submanifolds in space forms, Bull. Belg. Math. Soc. Simon Stevin 13 (2006), no. 4, 689-701. MR 2300625(2008c:53050)

4. D. Gromoll and K. Grove, A generalization of Berger's rigidity theorem for positively curved manifolds, Ann. Sci. Ë̈ole Norm. Sup. 11 (1987), 227-239. MR911756 (88k:53062)

5. D. Gromoll and K. Grove, The low-dimensional metric foliations of Euclidean spheres, J. Diff. Geom. 28 (1988), 143-156. MR950559 (89g:53052)

6. D. Gromoll and G. Walschap, The metric fibrations of Euclidean space, J. Diff. Geom. 57 (2001), 233-238. MR.1879226 (2002k:53053)

7. K. Grove, Geometry of, and via, symmetries, in Conformal, Riemannian and Lagranian geometry, the 2000 Barrett Lectures, University Lecture Series, Vol. 27, Amer. Math. Soc., Providence, RI, 2002. MR 1922721 (2003g:53046)

8. D. Hilbert and S. Cohn-Vollsen, Geometry and the imagination, Chelsea Publishing Company, New York, NY, 1952. MR0046650(13:766c)

9. M. Kerin and K. Shankar, Riemannian submersions from simple compact Lie groups, preprint, 2009.

10. M. Munteanu, One-dimensional metric foliations on compact Lie groups, Michigan Math. J. 54 (2006), 25-32. MR2214786 (2007i:53025)

11. A. Ranjan, Riemannian submersions of compact simple Lie groups with connected totally geodesic fibers, Math. Z. 191, no. 2 (1986), 239-246. MR818668 (87d:53093)

12. K. Tapp, Nonnegatively curved vector bundles with large normal holonomy groups, Proc. Amer. Math. Soc. 136 (2008), no. 1, 295-300. MR2350416 (2008h:53084)

13. K. Tapp, Flats in Riemannian submersions from Lie groups, Asian J. Math., 13 (2009), no. 4, 459-464. MR2653712

14. B. Wilking, Index parity of closed geodesics and rigidity of Hopf fibrations, Invent. Math. 144 (2001), 281-295. MR1826371 (2002k:53042)

15. B. Wilking, A duality theorem for Riemannian foliations in nonnegative sectional curvature, Geom. Funct. Anal. 17 (2007), no. 4, 1297-1320. MR2373019 (2009a:53047)

Department of Mathematics, Computer Science, and Statistcs, SUny Oneonta, ONEONTA, NEW YoRK 13820

E-mail address: munteam@oneonta.edu

Department of Mathematics, Saint Joseph's University, 5600 City Avenue, PhiladelPhia, Pennsylvannia 19131

E-mail address: ktapp@sju.edu 